\title{
Geometry of Invariant Tori of Certain Integrable Systems with Symmetry and an Application to a Nonholonomic System ${ }^{\star}$
}

\author{
Francesco FASSÒ and Andrea GIACOBBE \\ Dipartimento di Matematica Pura e Applicata, Università di Padova, \\ Via Trieste 63, 35131 Padova, Italy \\ E-mail:fasso@math.unipd.it, giacobbe@math.unipd.it
}

Received November 20, 2006, in final form March 15, 2007; Published online March 22, 2007

Original article is available at http://www.emis.de/journals/SIGMA/2007/051/

\begin{abstract}
Bifibrations, in symplectic geometry called also dual pairs, play a relevant role in the theory of superintegrable Hamiltonian systems. We prove the existence of an analogous bifibrated geometry in dynamical systems with a symmetry group such that the reduced dynamics is periodic. The integrability of such systems has been proven by M. Field and J. Hermans with a reconstruction technique. We apply the result to the nonholonomic system of a ball rolling on a surface of revolution.
\end{abstract}

Key words: systems with symmetry; reconstruction; integrable systems; nonholonomic systems

2000 Mathematics Subject Classification: 37J35; 70H33

\section{Introduction}

A number of nonholonomic mechanical systems are integrable, in the sense that their motions are conjugate to a linear flow on the torus, namely, are quasiperiodic. A noteworthy example is a heavy sphere which rolls without sliding inside a convex surface of revolution with vertical symmetry axis. This system has symmetry group $\mathrm{SO}(3) \times S^{1}$ and the reduced system was known to be integrable already to Routh [18], who showed that the equations of motion can be solved by quadratures depending on the solution of a linear ODE. The quasiperiodicity of the unreduced system was proven much more recently by J. Hermans [13] (see also [19]) by means of a remarkable reconstruction argument.

In fact, it has been shown by M. Field [10] and J. Hermans [13] that, if a dynamical system is invariant under the (free) action of a compact Lie group and if the reduced dynamics is periodic, then the reconstructed dynamics is quasi-periodic. Specifically, there is a fibration of a certain 'regular' subset of the phase space by invariant tori of dimension $r+1$, where $r$ is the rank of the group ${ }^{1}$, on which the flow is conjugate to a linear flow. Moreover, the frequencies of these motions depend only on the integrals of motion of the reduced system. As proven by N.T. Zung in [20], a stronger result is true in the Hamiltonian setting, where quasi-periodicity of the reconstructed flow follows also from quasi-periodicity of the reduced flow.

The purpose of this paper is to investigate the global geometry of the fibration by the invariant tori of these systems, with the analogy of the Hamiltonian case in mind. Integrability of Hamiltonian systems is usually related to the existence of integrals of motion with certain properties via the Liouville-Arnol'd theorem and its 'noncommutative' generalizations (see e.g. [14, 7]

${ }^{\star}$ This paper is a contribution to the Proceedings of the Workshop on Geometric Aspects of Integrable Systems (July 17-19, 2006, University of Coimbra, Portugal). The full collection is available at http://www.emis.de/journals/SIGMA/Coimbra2006.html

${ }^{1}$ Some notions on Lie groups are collected in the Appendix. 
for reviews and further references). The resulting structure is characterized by the existence of not just one, but two invariant foliations of the phase space, which are naturally defined and interrelated: the fibration by the (isotropic) invariant tori and its (coisotropic) 'polar' foliation, which together form what in symplectic geometry is called a 'dual pair'. For our purposes, it is especially useful to describe this structure in the case in which the integrability is related to the existence of a symmetry group (see e.g. [17, 11, 1] for details, extensions, and further references). Assume that the Hamiltonian is invariant under the action of a compact and connected Lie group $G$, which acts freely and in a Hamiltonian way on the phase space $M$. Assume moreover, just for simplicity, that the momentum map $J: M \rightarrow \mathfrak{g}$ has connected fibers. If $\operatorname{dim} M=\operatorname{dim} G+\operatorname{rank} G$, then

- The fibers of the momentum map $J: M \rightarrow \mathfrak{g}^{*}$ are the orbits of a maximal torus of $G$ and hence are diffeomorphic to $\mathbb{T}^{r}$, where $r=\operatorname{rank} G$. They are isotropic, and the dynamics is conjugate to a linear flow on them.

- Globally, the momentum map $J: M \rightarrow B:=J(M) \subset \mathfrak{g}^{*}$ is a fibration with fiber $\mathbb{T}^{r}$.

- The orbits of $G$, being symplectically orthogonal to the level sets of $J$, are coisotropic and union of invariant tori. More precisely, each $G$-orbit is a $\mathbb{T}^{r}$-bundle over a regular coadjoint orbit of $G$.

- Globally, the $G$-orbits are the fibers of a fibration $\pi_{A}: M \rightarrow A$, where $A$ is a manifold of dimension equal to rank $G$ which can be indentified with a Weyl chamber of $\mathfrak{g}$. The components of $\pi_{A}$ play the role of the 'actions' of the system.

- The two fibrations are compatible, in the sense that $\pi_{A}=\rho \circ J$, where $\rho: \mathfrak{g}^{*} \rightarrow A$ gives the foliation of $\mathfrak{g}^{*}$ into its coadjoint orbits.

Sometimes, the Hamiltonian is independent of the momentum map and the energy-momentum map $(H, J)$ turns out to be the momentum map of a $G \times S^{1}$-action (see noticeably [1] chapter 12). In these cases, the previous picture applies with $G$ replaced by $G \times S^{1}$ and $r$ replaced by $r+1$. This is the situation that most directly compares to the case treated in the sequel.

The above 'bifibrated' structure, which plays an important role in a number of questions, see e.g. [14, 7], can be described via the existence of a commutative diagram such as in Fig. 1 left. Fig. 1 right gives a pictorial representation of this structure, see e.g. [7], where the individual flowers are the coisotropic leaves, diffeomorphic to $G$, the petals are the invariant tori $\mathbb{T}^{r}$, the centers of the flowers are the coadjoint orbits of $\mathfrak{g}^{*}$, and the meadow is a Weyl chamber, namely, the 'action space'. The picture is inaccurate in that it suggests that both fibrations $J$ and $\pi_{A}$ are topologically trivial, while it is not necessarily so.
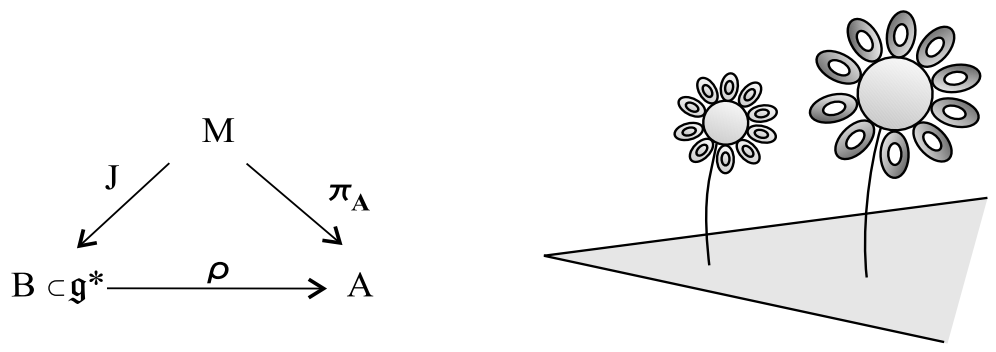

Figure 1. The bifibration (left) and its pictorial representation (right).

In this paper, extending previous results by Hermans that we review in Section 2, we show that a bifibrated structure is encountered also in the case of systems whose integrability is determined via reconstruction from a periodic reduced dynamics (Section 3). The difference, of course, is that there is no symplectic interpretation of the 'polarity' between the two fibrations. In Section 4 we relate the integrability to Bogoyavlenskij's integrability [2] and study the integrals 
of motion produced by the reconstruction procedure. Finally, in Section 5 we illustrate the previous constructions on the example, already considered by Hermans, of the sphere inside a convex surface of revolution.

While preparing this paper, we were informed by Richard Cushman that a very similar extension of Hermans' work will appear in [5]. The main difference, to our understanding, is that we focus on the bifibrated structure, while [5] emphazises non-compact groups and non-free actions.

\section{Reconstruction of the invariant tori}

A. The phase map. For a symmetric system with periodic reduced dynamics the reconstruction of the unreduced dynamics is a well known topic, see e.g. [15], and requires introduction of a Lie-group valued map called phase. Consider:

H1. A free action $\Psi: G \times M \rightarrow M$ of a compact connected Lie group $G$ on a manifold $M$.

Since the group is compact, freeness of the action implies properness, so the quotient space $\bar{M}:=M / G$ is a manifold. Moreover, the quotient map $\pi: M \rightarrow \bar{M}$ defines a $G$-principal bundle. We often write $\Psi_{g}(m)$ or $g . m$ for $\Psi(g, m)$ with $g \in G$ and $m \in M$. Next, we consider

H2. A G-invariant vector field $X$ on $M$.

If we denote by $\Phi_{t}^{X}$ the map at time $t$ of the flow of $X$, then $\Psi_{g} \circ \Phi_{t}^{X}=\Phi_{t}^{X} \circ \Psi_{g}$ for all $t \in \mathbb{R}$ and $g \in G$. The flow of the reduced vector field $\bar{X}:=\pi_{*} X$ on $\bar{M}$ is intertwined by $\pi$ with that of $X$, that is, $\pi \circ \Phi_{t}^{X}=\Phi_{t}^{\bar{X}} \circ \pi$. We further assume that:

H3. The reduced vector field $\bar{X}$ has periodic flow, with positive smooth (minimal) period.

Here, smoothness of the period means that the function $\bar{\tau}: \bar{M} \rightarrow \mathbb{R}$, which associates to each point $\bar{m} \in \bar{M}$ the minimal period $\bar{\tau}(\bar{m})$ of the $\bar{X}$-orbit through it, is smooth. The function $\bar{\tau}$ defines a function $\tau=\bar{\tau} \circ \pi$ on $M$.

Hypothesis $\mathrm{H} 3$ also implies that the orbit space of $\bar{X}$, that we denote $A$, is a smooth manifold, and that the quotient map $\bar{\pi}: \bar{M} \rightarrow A$ defines a $S^{1}$-principal bundle, see [8]. We denote $\pi_{A}$ the function $\bar{\pi} \circ \pi: M \rightarrow A$, as in the following diagram:

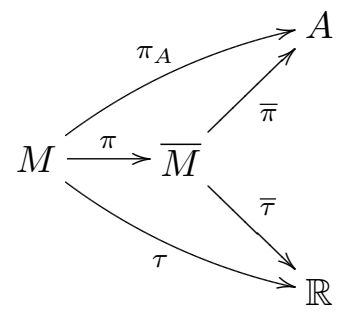

The definition of the phase map is based on the fact that the periodicity of the reduced dynamics implies that the unreduced orbit of a point $m$ in $M$ returns periodically, with period $\tau(m)$, to the $G$-orbit through $m$, see Fig. 2. Therefore, there exists an element $\gamma(m) \in G$ (unique, since the action is free) such that

$$
\Phi_{\tau(m)}^{X}(m)=\Psi_{\gamma(m)}(m) .
$$

This defines the phase map $\gamma: M \rightarrow G$. This map is called monodromy in [13].

In reconstruction theory, where $\gamma(m)$ is usually called total phase or reconstruction phase, the emphasis is often on computation and properties of the individual reconstructed orbits. A different approach has been pursued by M. Field [10] and by J. Hermans [13] who used this map, together with the periodicity of the reduced flow, to prove the quasi-periodicity of the unreduced dynamics. A central role in this approach is played by the fact that the phase map is a conjugacy-equivariant $G$-valued integral of motion of the unreduced dynamics: 
Proposition 1. Under hypotheses $\mathrm{H} 1-\mathrm{H} 3$, the phase map $\gamma: M \rightarrow G$ is

(i) smooth;

(ii) constant along the $X$-orbits, that is $\gamma \circ \Phi_{t}^{X}=\gamma$ for all $t$;

(iii) equivariant with respect to the group conjugacy, that is $\gamma(g . m)=g \gamma(m) g^{-1}$ for all $g \in G$ and $m \in M$.

Proof. Consider the manifold $\mathcal{M}=\{(m, g . m) \mid m \in M \quad g \in G\} \subset M \times M$ and the submersion $f: M \times G \rightarrow \mathcal{M}$ defined by $(m, g) \mapsto(m, g \cdot m)$. Since $\mathcal{N}=\left\{\left(m, \Phi_{\tau(m)}^{X}(m) \mid m \in M\right\}\right.$ is a smooth submanifold of $\mathcal{M}$ of codimension $\operatorname{dim} G$, the implicit function theorem together with the fact that projection onto $M$ of $f^{-1}(\mathcal{N})$ is $1: 1$ implies that $f^{-1}(\mathcal{N})$ is locally the graph of a smooth function $\gamma: M \rightarrow G$.

Statements (ii) and (iii) follow from the facts that the period function $\tau$ is constant along the orbits of $X$ and along the orbits of $G$, that is $\tau(m)=\tau\left(\Phi_{t}^{X}(m)\right)=\tau(g . m)$ for all $g \in G$ and $t \in \mathbb{R}$. It follows that $\Phi_{\tau(m)}^{X}\left(\Phi_{t}^{X}(m)\right)=\Phi_{t}^{X}\left(\Phi_{\tau(m)}^{X}(m)\right)=\Phi_{t}^{X}(\gamma(m) \cdot m)=\gamma(m) \cdot\left(\Phi_{t}^{X}(m)\right)$, hence $\gamma\left(\Phi_{t}^{X}(m)\right)=\gamma(m)$, and that $\Phi_{\tau(m)}^{X}(g \cdot m)=g \cdot \Phi_{\tau(m)}^{X}(m)=g \cdot \gamma(m) \cdot m=\left(g \gamma(m) g^{-1}\right)(g \cdot m)$, hence $\gamma(g . m)=g \gamma(m) g^{-1}$.

B. Invariant tori. Let $Z(h)$ be the centralizer of $h \in G$. As is recalled in the Appendix, for a dense and open set of points $h \in G$, called regular points, $Z(h)$ is a torus contained in $G$ of maximal dimension $r$. We denote $M_{\text {reg }}$ the set of points $m \in M$ whose phase $\gamma(m)$ is a regular element of $G$.

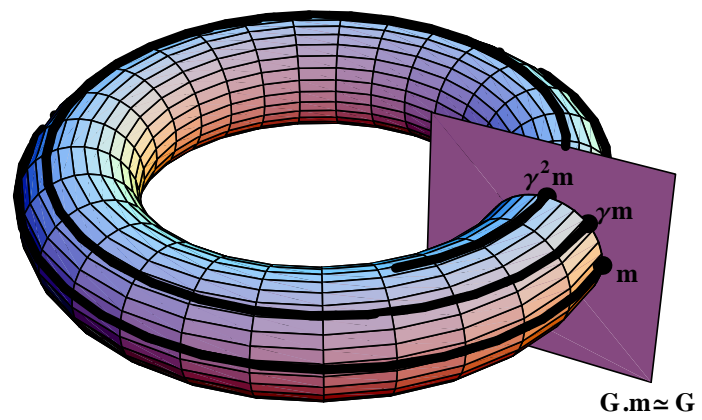

Figure 2. The $T_{m}$-orbit of the centralizers of $\gamma(m)$ (the circle) in the $G$-orbit (the shaded plane), and its transport along the flow of $X$ (the torus).

For $m \in M_{\text {reg }}$, the maximal torus $T_{m}:=Z(\gamma(m))$, and hence the set $T_{m} . m$, is diffeomorphic to $\mathbb{T}^{r}=(\mathbb{R} / \mathbb{Z})^{r}$. Since all the elements of $T_{m} \cdot m$ have the same phase, and since $T_{m} \cdot m$ is mapped onto itself by the map $\Phi_{\tau(m)}^{X}$, one can define a foliation of $M_{\text {reg }}$ by $X$-invariant tori of dimension $r+1$ by transporting the $T_{m}$-orbits along the flow of $X$, see Fig. 2. More precisely, following $[10,13]$, we have:

Proposition 2. Consider a point $m \in M_{\mathrm{reg}}$. Let $\eta_{m}$ be an element in the Lie algebra $\mathfrak{t}_{m}$ of $T_{m}$ such that $\gamma(m)=\exp \eta_{m}$. Define the map

$$
j_{m}: S^{1} \times T_{m} \rightarrow M_{\mathrm{reg}}, \quad j_{m}(\alpha, h)=\Psi_{h} \circ \Phi_{\alpha \tau(m)}^{X} \circ \Psi_{\exp \left(\alpha \eta_{m}\right)}^{-1}(m) .
$$

(i) The map $j_{m}$ is an embedding and its image $P_{m}:=j_{m}\left(S^{1} \times T_{m}\right)$ is invariant under the flow of $X$ and diffeomorphic to $\mathbb{T}^{r+1}$.

(ii) If $m^{\prime}$ belongs to $P_{m}$ then $P_{m^{\prime}}=P_{m}$.

Proof. (i) Since $S^{1} \times T_{m}$ is compact we need only to show that $j_{m}$ is an injective immersion. To prove that the map is an immersion consider a vector $(a, \xi) \in T_{(\bar{\alpha}, \bar{h})}\left(S^{1} \times T_{m}\right)$, with $a \in \mathbb{R}$ 
and $\xi \in \mathfrak{t}_{m}$. Such a vector can be represented by the curve $t \mapsto(\bar{\alpha}+t a, \exp (t \xi) \bar{h})$ whose image via $j_{m}$ is $t \mapsto \Psi_{\exp (t \xi) \bar{h}} \circ \Phi_{(\bar{\alpha}+t a) \tau(m)}^{X} \circ \Psi_{\exp ((\bar{\alpha}+t a) \eta)}^{-1}(m)$. By differentiation with respect to $t$, one sees that $(a, \xi)$ is mapped onto $X_{\xi}+a \tau(m) X-a X_{\eta}$, with $X_{\xi}, X_{\eta}$ the infinitesimal group action associated to the elements $\xi, \eta$. This vector vanishes if and only if $a=0$ and $\xi=0$.

The injectivity follows from the fact that if $j_{m}(\alpha, h)=j_{m}(\beta, k)$ then $\Phi_{(\alpha-\beta) \tau(m)}^{X}(m)=$ $\Psi_{h^{-1} \exp ((\alpha-\beta) \eta) k}(m)$. The left hand-side belongs to G.m if and only if $n=\alpha-\beta \in \mathbb{Z}$, in which case the equation becomes $\gamma(m)^{n} \cdot m=h^{-1} \gamma(m)^{n} k \cdot m$. This equation holds if and only if $h=k$.

The invariance of $P_{m}$ under the flow of $X$ is obvious. In fact if $m^{\prime}=j_{m}(\alpha, h)$, then $t \mapsto$ $j_{m}(\alpha+t, \exp (t \eta(m)) h)$ is the flow of $X$ through $m^{\prime}$.

(ii) Let $m^{\prime}=j_{m}(\alpha, h) \in P_{m}$ for some $\alpha \in S^{1}$ and $h \in T_{m}$, hence $m=j_{m^{\prime}}\left(-\alpha, h^{-1}\right)$. If $m^{\prime \prime} \in P_{m}$, then $m^{\prime \prime}=j_{m}(\beta, k)$ and hence $m^{\prime \prime}=j_{m^{\prime}}\left(k h^{-1}, \beta-\alpha\right) \in P_{m^{\prime}}$.

We shall call the sets $P_{m}$ either 'invariant tori of $X$ ' or, more frequently, petals. Note that while, for given $m$, the embedding $j_{m}$ depends on the choice of the element $\eta_{m}$ in the algebra, the petal through it depends only on $X$ and on the $G$-action.

Item ii. in the previous Proposition implies that the petals give a partition of the manifold $M_{\text {reg. }}$. However, since in every sufficiently small open set $U \subset M_{\text {reg }}$ one can choose $\eta_{m}$ as a smooth function of $m$, the argument used in the proof of the Proposition yields a $\mathbb{T}^{r+1}$-action on $U$ whose orbits are the petals $P_{m}, m \in U$. This implies that the partition of $M_{\text {reg }}$ in petals is a foliation. We do not detail this construction here since we shall later show that the petals are in fact the fibers of a (global) fibration.

C. Quasi-periodicity of the flow. Following [13], we now show that the flow of $X$ on each petal $P_{m}$ is quasi-periodic, that is, it is conjugate to the linear flow on $\mathbb{T}^{r+1}$. To this end we introduce suitable coordinates on the maximal torus $T_{m}$. Specifically, choose a basis $\xi_{1}, \ldots, \xi_{r}$ of the Lie algebra $\mathfrak{t}_{m}$ of $T_{m}$ that generates the lattice of elements of $\mathfrak{t}_{m}$ that exponentiate to the identity. The map $\Xi:\left(\beta_{1}, \ldots, \beta_{r}\right) \mapsto \exp \left(\sum_{j} \beta_{j} \xi_{j}\right)$ is a diffeomorphism from $\mathbb{T}^{r}$ onto $T_{m}$ and

$$
i_{m}:=j_{m} \circ(\mathrm{id} \times \Xi): S^{1} \times \mathbb{T}^{r} \rightarrow M_{\mathrm{reg}}
$$

is an embedding of $\mathbb{T}^{r+1}$ into $M_{\text {reg. }}$. Explicitly, $i_{m}(\alpha, \beta)=\Psi_{\Xi(\beta)} \circ \Phi_{\alpha \tau(m)}^{X} \circ \Psi_{\exp (\alpha \eta)}^{-1}(m)$.

Proposition 3. Consider $m \in M_{\mathrm{reg}}$ and let $\gamma(m)=\exp \eta$ with $\eta=\sum_{j} \eta_{j} \xi_{j}$. The map $i_{m}$ intertwines the flow of $X$ and the linear flow on $\mathbb{T}^{r+1}$ given by

$$
\varphi_{t}(\alpha, \beta):=\left(\alpha+\frac{t}{\tau(m)}, \beta+\frac{t}{\tau(m)} \vec{\eta}\right) \quad(\bmod 1)
$$

where $\vec{\eta}=\left(\eta_{1}, \ldots, \eta_{r}\right)$.

Proof. This is a simple computation, let $\tau=\tau(m)$ :

$$
\begin{aligned}
i_{m}\left(\varphi_{t}(\alpha, \beta)\right) & =\Psi_{\left(\Xi\left(\beta+\frac{t}{\tau} \vec{\eta}\right)\right)} \circ \Phi_{\left(\alpha+\frac{t}{\tau}\right) \tau}^{X} \circ \Psi_{\exp \left(\left(\alpha+\frac{t}{\tau}\right) \eta\right)}^{-1}(m) \\
& =\Psi_{\Xi(\beta)} \circ \Psi_{\exp \left(\frac{t}{\tau} \eta\right)} \circ \Phi_{\alpha \tau}^{X} \circ \Phi_{t}^{X} \circ \Psi_{\exp (\alpha \eta)}^{-1} \circ \Psi_{\exp \left(\frac{t}{\tau} \eta\right)}^{-1}(m) \\
& =\Phi_{t}^{X} \circ \Psi_{\Xi(\beta)} \circ \Phi_{\alpha \tau}^{X} \circ \Psi_{\exp (\alpha \eta)}^{-1}(m) \\
& =\Phi_{t}^{X}\left(i_{m}(\alpha, \beta)\right),
\end{aligned}
$$

where we have used the commutativity of $X$-flow and $T$-action and the fact that the latter is Abelian. 


\section{The bifibration}

A. The flower fibration. Since the dynamics in two petals $P_{m}$ and $P_{g \cdot m}=g \cdot P_{m}$ is conjugate by $g$, it is quite natural to group the petals to form bigger sets of $G$-related petals

$$
F_{m}:=G \cdot P_{m}, \quad m \in M_{\mathrm{reg}},
$$

that we call flowers. A hint about the relevance of this further structure can be found in [12], where however it is not exploited.

We begin the study of this structure by characterizing the 'internal' structure of each flower, that is, its quotient over the petals. Note that, by Proposition 1, each flower is contained in $M_{\text {reg }}$ and the maximal tori of its points are all conjugate.

Proposition 4. Consider $m \in M_{\mathrm{reg}}$ and let $T_{m}$ be the maximal torus $Z(\gamma(m))$. Then, $F_{m}$ is diffeomorphic to $S^{1} \times G$ and is a $\mathbb{T}^{r+1}$-principal bundle with the petals as fibers and base $G / T_{m}$.

Proof. The proof that $F_{m} \cong S^{1} \times G$ is very similar to the proof of Proposition 2. Fix $m \in M_{\text {reg }}$ and choose $\eta_{m}$ such that $\exp \left(\eta_{m}\right)=\gamma(m)$. Consider the map

$$
J_{m}: S^{1} \times G \rightarrow M, \quad(\alpha, g) \mapsto \Psi_{g} \circ \Phi_{\alpha \tau(m)}^{X} \circ \Psi_{\exp \left(\alpha \eta_{m}\right)}^{-1}(m) .
$$

For the reasons given in Proposition 2 this map is an embedding, so its image is diffeomorphic to $S^{1} \times G$. That this image is $F_{m}$ follows from the fact that $F_{m}=\bigcup_{0 \leq t<\tau(m)} \Phi_{t}^{X}(G . m)$.

The smooth map

$$
\chi: S^{1} \times T_{m} \times F_{m} \rightarrow F_{m}, \quad \chi\left(a, b, J_{m}(\alpha, g)\right)=J_{m}(a+\alpha, g b)
$$

gives a free action of $\mathbb{T}^{r+1}$ on $F_{m}$, which is therefore a $\mathbb{T}^{r+1}$-principal bundle. The orbits of this action are the sets $g . P_{m}$, with $g \in G$. Observing that $J_{m}$ pulls back each set $g . P_{m}$ to the set $S^{1} \times g T_{m}$ in $S^{1} \times G$, one concludes that the quotient of $F_{m}$ by the petals is diffeomorphic to the quotient of $S^{1} \times G$ by $S^{1} \times T_{m}$.

B. The petal-flower bifibration. Proposition 4 implies also that the flowers are the fibers of a fibration of $M_{\text {reg. }}$. In fact, they are the level sets of the map

$$
\pi_{A}: M_{\text {reg }} \rightarrow A_{\text {reg }}:=\pi_{A}\left(M_{\text {reg }}\right) \subset A
$$

and, by the Ehresmann fibration theorem, any submersion with compact and connected fibers is a locally trivial fibration, see [16]. We now prove that also the petals are the fibers of a fibration of $M_{\mathrm{reg}}$, thus obtaining the following bifibrated structure:

Theorem 1. There exists a manifold $B$ and a commutative diagram

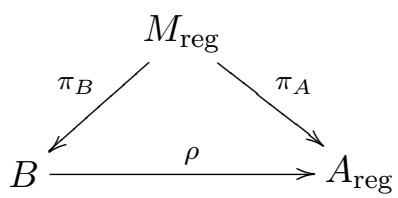

where:

- the $\operatorname{map}_{B}$ is a fibration whose fibers are the petals, diffeomorphic to $\mathbb{T}^{r+1}$;

- the level sets of the fibration $\pi_{A}$ are the flowers, diffeomorphic to $S^{1} \times G$;

- the level sets of $\rho$ are the the quotient space flowers/petals and are diffeomorphic to $G / T$, where $T$ is any chosen maximal torus of $G$.

The dynamical system evolves in the petals, and is there a linear flow. 
The remainder of this section is devoted to the proof of this Theorem, which is the main result of the paper. In [13], it is proven that the petals form the fibers of semilocal fibrations, where semilocal means in a neighbourhood of a petal.

C. The semiglobal normal form. The proof of Theorem 1 is rather technical and is articulated in two steps. First we describe the semiglobal geometry of the fibration by petals, where semiglobal means in a $G$-invariant neighbourhood of each flower, not just in a neighbourhood of a petal. We do so by defining a sort of semiglobal normal form for the bifibration in petals and flowers. The argument we use is a direct extension of that of Hermans [13], who essentially proves the same result semilocally. In the next subsection we shall construct the base $B$ of the fibration by petals with a cut and paste construction.

Lemma 1. For every $m \in M_{\text {reg }}$ there is an open neighbourhood $U$ of $\pi_{A}(m)$ in $A_{\text {reg }}$, a discrete $\mathbb{Z}$-action on $G \times U \times \mathbb{R}$ and a diffeomorphism $\iota_{U}: M_{U}:=\pi_{A}^{-1}(U) \rightarrow(G \times U \times \mathbb{R}) / \mathbb{Z}$ which intertwines:

- the flow of $X$ in $M_{U}$ and the map $t^{\prime} \mapsto\left[g, u, t+t^{\prime}\right]$ on $(G \times U \times \mathbb{R}) / \mathbb{Z}$;

- the action of $G$ on $M_{U}$ and the $G$-action $g^{\prime} \cdot[g, u, t]=\left[g^{\prime} g, u, t\right]$ on $(G \times U \times \mathbb{R}) / \mathbb{Z}$, where the square brackets represent the equivalence classes.

The proof of this Lemma rests upon another, technical, Lemma:

Lemma 2. Given any maximal torus $T$ of $G$ and any $a \in A_{\text {reg, }}$, there exists an open neighbourhood $U$ of a and a section $\sigma: U \rightarrow M_{\mathrm{reg}}$ of $\pi_{A}$ such that the phase map $\gamma \circ \sigma$ has values in $T$.

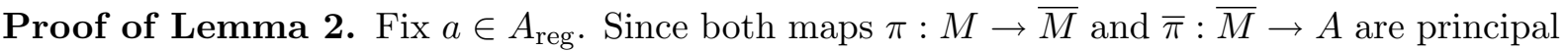
bundles there is a neighbourhood $U \subset A_{\text {reg }}$ of $a$ and a section $\sigma^{\prime}: U \rightarrow M_{\text {reg }}$ of $\pi_{A}$. Let $m=\sigma^{\prime}(a)$. The element $\gamma(m)$ belongs to $G$ and necessarily there exists an element $g \in G$ such that $\gamma(g . m)=g \gamma(m) g^{-1}$ belongs to $T$ (see the Appendix). It follows that the section $\sigma^{\prime \prime}=g . \sigma^{\prime}$ maps $a$ to the element $g . m$ such that the phase $\gamma(g . m) \in T$.

The Lie algebra $\mathfrak{g}$ of $G$ can be decomposed into $\mathfrak{t} \oplus V$, where $\mathfrak{t}$ is the Lie algebra of $T$ and $V$ is a subspace of $\mathfrak{g}$. Consider the map

$$
f: V \times U \longrightarrow G, \quad(\xi, u) \mapsto \operatorname{Ad}_{\exp (\xi)} \gamma\left(\sigma^{\prime \prime}(u)\right) .
$$

Observe that $f(0, a)$ belongs to $T$, that $T$ is a submanifold of $G$ of codimension equal to the dimension of $V$, and that $d_{(0, a)} f(V \times 0)$ is a subspace of the tangent space $T_{\gamma\left(\sigma^{\prime \prime}(a)\right)} G$ transversal to $T_{\gamma\left(\sigma^{\prime \prime}(a)\right)} T$. The implicit function theorem implies that possibly restricting $U, f^{-1}(T)$ is the graph of a smooth function $\xi: U \rightarrow V$ such that $\xi(a)=0$. This allows the definition of a section $\sigma: u \mapsto \exp (\xi(u)) \sigma^{\prime \prime}(u)$, which has the required property.

Proof of Lemma 1. Fix a maximal torus $T$ of $G$. There is an open covering $\mathcal{U}$ of $A_{\text {reg }}$ by open sets $U$ as in Lemma 2, namely, for each $U \in \mathcal{U}$ there is a section $\sigma_{U}$ of $\pi_{A}$ such that $\gamma \circ \sigma_{U}(U) \subset T$. Define the map $\iota_{U}: G \times U \times \mathbb{R} \rightarrow M_{\mathrm{reg}},(g, u, t) \mapsto \Phi_{t}^{X}\left(g \cdot \sigma_{U}(u)\right)$. This map is easily shown to be an immersion, invariant under the action of $G$, and invariant under the flow of $X$. Consider an element $y$ in the image of $\iota_{U}$ and a triplet $(g, u, t)$ such that $\iota_{U}(g, u, t)=y$. The set of elements $\left(g^{\prime}, u^{\prime}, t^{\prime}\right)$ such that $\iota_{U}\left(g^{\prime}, u^{\prime}, t^{\prime}\right)=y$ is the set $\left\{\left(g \gamma\left(\sigma_{U}(u)\right)^{-n}, u, t+n \bar{\tau}(u)\right) \mid n \in \mathbb{Z}\right\}$. We hence define the $\mathbb{Z}$-action $n \cdot(g, u, t)=\left(g \gamma\left(\sigma_{U}(u)\right)^{-n}, u, t+n \bar{\tau}(u)\right)$. The quotient of $G \times U \times \mathbb{R}$ under this $\mathbb{Z}$-action is diffeomorphic to the image of $\iota_{U}$, that is the open set $M_{U}=\pi_{A}^{-1}(U)$.

In each open set $M_{U}$ the map $\iota_{U}$ conjugates the flow of $X$ to the $\mathbb{R}$-action $t^{\prime}[g, s, t]=\left[g, s, t+t^{\prime}\right]$ and the $G$-action to $g^{\prime}[g, s, t]=\left[g^{\prime} g, s, t\right]$.

We call the diffeomorphism $\iota_{U}$ between $M_{U}$ and $(G \times U \times \mathbb{R}) / \mathbb{Z}$ a semiglobal normal form for $M$. 
The proof of Lemma 1 shows also that the flow of $X$ is contained in the sets $\iota_{U}(g T \times u \times \mathbb{R})$, which are the petals $P_{\iota_{U}(g, u, 0)}$, and in the bigger sets $\iota_{U}(G \times u \times \mathbb{R})$, which are the flowers $F_{\iota_{U}(g, u, 0)}$. We can thus write the semiglobal commutative diagrams
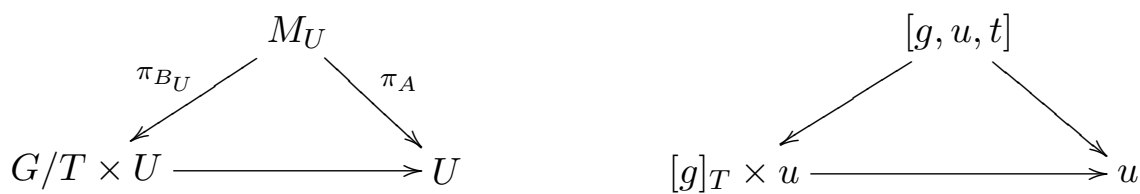

where $[g]_{T}$ stands for the class $g T$ in $G / T$. The fibers of $\pi_{B_{U}}$ are the petals, the fibers of $\pi_{A}$ are the flowers. The quotients obtained identifying the petals of a flower to points are all diffeomorphic to $G / T$. We call such manifolds centers.

Proof of Theorem 1. In order to prove Theorem 1, we now globalize the above construction of the maps $\pi_{B_{U}}: M_{U} \rightarrow G / T \times U$ so as to define a manifold $B$ and a map $\pi_{B}: M_{\text {reg }} \rightarrow B$. The construction of $B$ requires the preliminary construction of a certain covering space $\widetilde{A}$ of $A_{\text {reg }}$ by means of a cut and paste construction that we now describe.

Let $\mathcal{U}$ be a covering of $A_{\text {reg }}$ by open sets $U$ as in Lemma 1 and let $W=\left\{[e]_{T}=\left[g_{0}\right]_{T},\left[g_{1}\right]_{T}, \ldots\right\}$ be the Weyl group $N(T) / T$ (see the Appendix). An open covering of $\widetilde{A}$ is made of the open sets $W \times U$ with $U \in \mathcal{U}$. The gluing map between $W \times U_{1}$ and $W \times U_{2}$ is given by imposing that $\left(\left[g_{1}\right]_{T}, u_{1}\right) \in W \times U_{1}$ equals $\left(\left[g_{2}\right]_{T}, u_{2}\right) \in W \times U_{2}$ if and only if $u_{1}=u_{2}$ and $P_{g_{1} . \sigma_{1}\left(u_{1}\right)}=P_{g_{2} . \sigma_{2}\left(u_{2}\right)}$. In the expression above, for each $i=1,2, \sigma_{i}$ is the section of $\pi_{A}$ over $U_{i}$ whose existence is stated in Lemma 1 and $g_{i}$ is any representative in $N(T)$ of the element $\left[g_{i}\right]_{T} \in N(T) / T$. Note that the choice of a different representative $g_{i} b, b \in T$, has no effects on the petals since $P_{g_{i} b . \sigma(u)}=P_{g_{i} . \sigma(u)}$. In conclusion, every element of $\widetilde{A}$ can be non-uniquely represented as a pair $\left(\left[g_{i}\right]_{T}, u\right)$ with $\left[g_{i}\right]_{T} \in W$ and $u \in U$. The topology of $\widetilde{A}$ is induced by the topology of the sets $W \times U$, the space is Hausdorff and paracompact because $A$ is Hausdorff and paracompact and $W$ is finite.

The Weyl group $W$ acts as deck transformations of $\widetilde{A}$. Given an element of $\widetilde{A}$ represented by $\left(\left[g_{i}\right]_{T}, u\right)$ in $W \times U$, the element $\left[g_{j}\right]_{T} \in W$ maps it to $\left(\left[g_{j} g_{i}\right]_{T}, u\right)$. The Weyl group also acts on the space $G / T$ by right-multiplication, $\left[g_{j}\right]_{T}[g]_{T}=\left[g g_{j}^{-1}\right]_{T}$. We can hence define the manifold $B=G / T \times_{W} \widetilde{A}$, where the fibered product is obtained by means of the anti-diagonal action $\left[g_{j}\right]_{T} \cdot\left([g]_{T},\left(\left[g_{i}\right]_{T}, u\right)\right)=\left(\left[g g_{j}^{-1}\right]_{T},\left(\left[g_{j} g_{i}\right]_{T}, u\right)\right)$. The space $B$ is a covering space of $G / N(T) \times A$ with fiber $W$ and is in turn covered by $G / T \times \widetilde{A}$ with fiber $W$. Its points can be non-uniquely represented by pairs $\left([g]_{T},\left(\left[g_{i}\right]_{T}, u\right)\right)$.

We now define the map $\pi_{B}: M_{\text {reg }} \rightarrow B$. For each $m \in M_{\text {reg }}$ there exists an open set $U \in \mathcal{U}$ and three elements $u \in U, g \in G$ and $t \in \mathbb{R}$ such that $m=\iota_{U}(g, u, t)$, see Lemma 1 . We thus define

$$
\pi_{B}(m):=\left([g]_{T},\left([e]_{T}, u\right)\right) \in G / T \times_{W} \widetilde{A},
$$

where $e$ is the identity of $G$. In order to prove that the map $\pi_{B}$ is well defined we need to show that, if the point $m$ is also represented by $\iota_{U^{\prime}}\left(g^{\prime}, u^{\prime}, t^{\prime}\right)$ with $u^{\prime} \in U^{\prime} \in \mathcal{U}$, then the element $\left(\left[g^{\prime}\right]_{T},\left([e]_{T}, u^{\prime}\right)\right)$ coincides with $\left([g]_{T},\left([e]_{T}, u\right)\right)$. Both sets of data satisfy

$$
m=\Phi_{t^{\prime}}^{X}\left(g^{\prime} \cdot \sigma^{\prime}\left(u^{\prime}\right)\right)=\Phi_{t}^{X}(g \cdot \sigma(u)),
$$

where $\sigma$ and $\sigma^{\prime}$ are the sections of Lemma 1. This implies that $\sigma^{\prime}\left(u^{\prime}\right)=\Phi_{t-t^{\prime}}^{X}\left(g^{\prime-1} g . \sigma(u)\right)$. This means that $\left(\left[g^{\prime-1} g\right]_{T}, u\right)$ and $\left([e]_{T}, u^{\prime}\right)$ represent the same point of $\widetilde{A}$. Hence $\left(\left[g^{\prime}\right]_{T},\left([e]_{T}, u^{\prime}\right)\right)=$ $\left(\left[g^{\prime}\right]_{T},\left(\left[g^{\prime-1} g\right]_{T}, u\right)\right)$. By using the definition of fibered product we finally conclude that

$$
\left(\left[g^{\prime}\right]_{T},\left(\left[g^{\prime-1} g\right]_{T}, u\right)\right)=\left(\left[g^{\prime}\right]_{T}\left[g^{-1} g^{\prime}\right]_{T}^{-1},\left[g^{-1} g^{\prime}\right]_{T}\left(\left[g^{\prime-1} g\right]_{T}, u\right)\right)=\left([g]_{T},\left([e]_{T}, u\right)\right) .
$$




\section{The phase map and the integrals of motion}

As pointed out in particular by Bogoyavlenskij [2], but see also [9], quasi-periodicity of a flow can be (semilocally) linked to the presence of a number of first integrals and of a complementary number of commuting dynamical symmetries which preserve these first integrals. Specifically, if on a manifold $M$ and for some $n<d=\operatorname{dim} M$ there are

- a submersion $F: M \rightarrow \mathbb{R}^{d-n}$ with compact and connected fibers, and

- $n$ everywhere linearly independent and pairwise commuting vector fields $Y_{1}, \ldots, Y_{n}$ which are tangent to the fibers of $F$,

then the fibers of $F$ are diffeomorphic to $\mathbb{T}^{n}$ and any vector field $X$ on $M$ such that

$$
L_{X} F_{i}=0 \quad \text { and } \quad\left[X, Y_{j}\right]=0, \quad i=1, \ldots, d-n, \quad j=1, \ldots, n,
$$

is conjugate to a constant vector field on $\mathbb{T}^{n}$.

In the setting of the previous sections, where $n=r+1$, the fibration $\pi_{B}: M_{\text {reg }} \rightarrow B$ ensures the existence of local sets of $\operatorname{dim} M-r-1$ functionally independent integrals of motion of $X$, which are obtained by lifting local coordinates on $B$. For what concerns the commuting dynamical symmetries, the vector field $X$ and the chosen maximal torus $T$ define a semiglobal group action of $T^{r+1}$ (see the proof of Proposition 4). This action can be non-global, due to the possible non-trivial monodromy of the torus bundle (see $[6,4]$ for the notion of monodromy). Nonetheless, the semiglobal torus action defines in every set $M_{U}$ a family of $r$ independent vector fields.

Going beyond these elementary considerations, we link now the integrals of motion to the phase map $\gamma$, which is a Lie group-valued conserved quantity. Clearly, the projection $\pi_{A}: M \rightarrow A$ gives rise to the semiglobal existence of sets of $\operatorname{dim} A$ functionally independent integrals of motion of $X$, which are obtained by lifting functions on $A$. We thus focus on the integrals of motion coming from $B$ but not from $A$.

In the semiglobal normal form of Lemma 1, the expression of all these integrals is immediate. Since the petals (which are the level sets of a full set of integrals of motion) correspond to subsets of the form $[g T, u, \mathbb{R}]$, the projection onto $u$ (the map $\pi_{A}$ ) gives the integrals obtained by lifting functions on $A$. The other integrals of motion can then be obtained via the map

$$
\delta_{U}: M_{U} \longrightarrow G / T, \quad[g, u, t] \mapsto[g] .
$$

In fact, two points $[g, u, t]$ and $\left[g^{\prime}, u^{\prime}, t^{\prime}\right]$ in $M_{U}$ belong to the same flower if and only if $u^{\prime}=u$. They belong to the same petal if and only if $u^{\prime}=u$ and $[g]_{T}=\left[g^{\prime}\right]_{T}$. Globalizing this construction, we have

Proposition 5. Fix any maximal torus $T$ in $G$ and let $N(T)$ be the normalizer of $T$ in $G$. Then, the integrals of motion that are not lifts of functions on $A_{\mathrm{reg}}$ via $\pi_{A}$ are lifts of functions on $G / N(T)$ via the map

$$
\delta: M_{\mathrm{reg}} \rightarrow G / N(T), \quad m \mapsto\left[g_{m}\right],
$$

where $g_{m} \in G$ is such that $g_{m}^{-1} \gamma(m) g_{m} \in T$. The level sets of this map consist of $|N(T) / T|$ petals.

Proof. Given $U \in \mathcal{U}$, take any $m \in \pi_{A}^{-1}(U) \subset M_{\text {reg }}$ and let $g_{m}$ be such that $g_{m}^{-1} \gamma(m) g_{m}$ belongs to $T$. Then, there exists an element $h \in N(T)$ and a time $t$ such that $\Phi_{t}^{X}\left(h g_{m}^{-1} \cdot m\right)=\sigma_{U}\left(\pi_{A}(m)\right)$. It follows that the point $m$ is represented in a semiglobal normal form of Lemma 1 by the triple $\left[g_{m} h^{-1}, \pi_{A}(m),-t\right]$. Hence, $\delta(m)=\left[g_{m} h^{-1}\right]_{T}$ in $G / T$. A further projection on $G / N(T)$ gives the element $\left[g_{m}\right]_{N(T)}$. 
Remark 1. The function $\delta$ described in Proposition 5 is obtained by composing the function $\delta_{U}$ in formula (2) with the projection $G / T \rightarrow G / N(T)$. This gives a map that has the same rank as $\delta$. The only difference is in the number of connected components of the level sets. This choice allows an easier definition of $\delta$.

Remark 2. The manifold $G / T$ is diffeomorphic to any regular coadjoint orbit, while $G / N(T)$ is diffeomorphic to a coadjoint orbit modulo the action of the Weyl group. This gives a very strict analogy with the case of a Hamiltonian system with a Hamiltonian group action we described in the Introduction.

\section{An example: the ball rolling on a surface of revolution}

We illustrate now the above construction in the example of a heavy sphere rolling without sliding inside a convex surface of revolution which has vertical axis and faces upward. This system has been considered by Hermans, who actually used it as motivation and exemplification of his reconstruction theory [13]. We add a global perspective to this. For general reference on this system see $[13,19,8]$ and references therein.

The phase space of the system is the eight-dimensional manifold $\mathbb{R}^{2} \times \mathbb{R}^{2} \times \mathrm{SO}(3) \times \mathbb{R} \ni$ $(a, \dot{a}, Q, w)$, where $a \in \mathbb{R}^{2}$ are Cartesian coordinates of the center of mass of the sphere, $Q \in$ $\mathrm{SO}(3)$ fixes the attitude of the sphere, and $w \in \mathbb{R}$ is the component of the angular velocity normal to the surface. The dynamical vector field of the system is invariant under the action of $G=S^{1} \times \mathrm{SO}(3)$ given by $(\vartheta, R) \cdot(a, \dot{a}, Q, w)=\left(S_{\vartheta} a, S_{\vartheta} \dot{a}, R Q, w\right)$, where $S_{\vartheta}$ is the rotation of angle $\vartheta$ about the vertical axis. This action is free on the submanifold $M=\left(\mathbb{R}^{4} \backslash\{0\}\right) \times \mathrm{SO}(3) \times \mathbb{R}$ and the four-dimensional quotient manifold $M / G$ is diffeomorphic to $\bar{M}=\left(R^{3} \backslash\{0\}\right) \times \mathbb{R}$, with projection $(a, \dot{a}, w) \mapsto(b(a, \dot{a}), w)$, where $b: \mathbb{R}^{4} \backslash\{0\} \rightarrow \mathbb{R}^{3} \backslash\{0\}$ is the Hopf fibration.

It was classically known that this system has three independent integrals of motion, the energy $H$ and two functions $J_{1}$ and $J_{2}$ which are sometimes referred to as Chaplygin integrals. These three integrals are $G$-invariant and hence descend to independent integrals of motion $\bar{H}$, $\bar{J}_{1}, \bar{J}_{2}$ of the reduced system $\bar{X}$ in the quotient manifold $\bar{M}$. Moreover, the level sets of $\bar{H}$, $\bar{J}_{1}, \bar{J}_{2}$ are compact, and hence are finite disjoint union of circles, see $[19,8]$. These circles are the orbits of the reduced dynamical system $\bar{X}$, which is therefore periodic. (This was also shown using a different approach in [13]). The continuity of the period is shown in [8]. Thus, the system fits in the setting of the previous sections and, since $S^{1} \times \mathrm{SO}(3)$ has rank two, the dynamics is quasi-periodic on three-dimensional tori, with frequencies which depend only on $H$, $J_{1}, J_{2}$ [13]. Thus, there are two additional integrals of motions, besides $H, J_{1}, J_{2}$.

A complete description of these integrals, and of the bifibration, faces a number of difficulties. First, determination of the phase map appears to be prohibitive even for given (and simple) profiles of the surface of revolution. Without it, it is obviously not possible to completely determine $M_{\text {reg }}$, the two base manifolds $B$ and $A_{\text {reg }}$, and the two additional integrals of motion as in Proposition 5. Moreover, since it is also difficult to establish whether the fibers of $\left(H, J_{1}, J_{2}\right)$ are connected, we do not know if $A=\pi_{A}(M)$ coincides with the image of $M$ under the map $\left(H, J_{1}, J_{2}\right)$ or if it is a covering space of this image. Nonetheless, something can be said about the structure of the bifibration in this case:

Proposition 6. The regular region $M_{\mathrm{reg}}$ of the phase space of the ball rolling on a surface of revolution admits a petal-flower bifibration in which:

- the petals are 3 -tori [13];

- the five-dimensional base $B$ is a 2-sheeted covering of the manifold $\mathbb{R} P^{2} \times A_{\text {reg }}$;

- the flowers are diffeomorphic to $\mathrm{SO}(3) \times \mathbb{T}^{2}$ and the fibration in petals of each flower is a $\mathbb{T}^{3}$-bundle over a 2-sphere, $\mathrm{SO}(3) \times \mathbb{T}^{2} \rightarrow S^{2}$, with projection the Hopf map from the $\mathrm{SO}(3)$-component onto $S^{2}$. 
Proof. The only fact we need to prove is the statement about the structure of $B$. Since the Weyl group is in this case $\mathbb{Z}_{2}, B=S^{2} \times_{\mathbb{Z}_{2}} \widetilde{A}_{\text {reg }}$ with $\widetilde{A}_{\text {reg }}$ a 2 -sheeted covering of $A_{\text {reg. }}$. Thus, $B$ is a two sheeted covering of $\mathbb{R} P^{2} \times A_{\text {reg. }}$.

Once we have partly clarified the structure of $B$, we may describe, up to the determination of $\gamma$ and some finite covering, the two additional functions of $B$ that are not lifts of functions of $A_{\text {reg. }}$. To this effect we need to define two independent functions on $\mathbb{R} P^{2}$, lift them to $B$, and

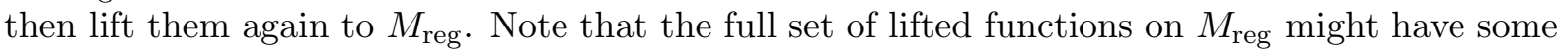
extra finite symmetry, in which case their levels will be finite families of petals.

We need to re-trace what was done in Proposition 5 in order to define the function $\delta$. Any point $(a, \dot{a}, Q, w)$ in $M$ has phase $\gamma(a, \dot{a}, Q, w)=(\vartheta(a, \dot{a}, Q, w), R(a, \dot{a}, Q, w))$, with $\vartheta$ a circlevalued function and $R$ an $S O(3)$-valued function. Following Proposition 5, we fix a maximal torus of $S^{1} \times S O(3)$ : we elect the torus $S^{1} \times S_{z}^{1}$, where $S_{z}^{1}$ are the rotations around the $z$-axes.

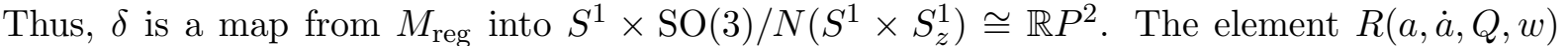
is a rotation $R_{\vec{v}}^{\varphi}$ of angle $\varphi$ about the oriented axis $\vec{v}$. A rotation that conjugates $R_{\vec{v}}^{\varphi}$ to $R_{\vec{e}_{3}}^{\varphi}$ is $R_{\vec{v} \times \vec{e}_{3}}^{\arccos \left(\vec{v}, \vec{e}_{3}\right)}$. It follows that $\gamma\left(R_{\vec{v} \times \vec{e}_{3}}^{\arccos \left(\vec{v}, \vec{e}_{3}\right)} \cdot(a, \dot{a}, Q, w)\right) \in S^{1} \times S_{z}^{1}$, and hence belongs to the maximal torus we have chosen: we have determined the element $g_{m}$ of Proposition 5 . We can hence state:

Proposition 7. The map $\delta$ has values in $\mathbb{R} P^{2}$ and is given by $(a, \dot{a}, Q, w) \mapsto\left[R_{\vec{v} \times \vec{e}_{3}}^{\arccos \left(\vec{v}, \vec{e}_{3}\right)} \vec{e}_{3}\right]$. (In this last expression, the square brackets denote the equivalence classes with respect to the projection $\mathbb{R}^{3} \backslash\{0\} \rightarrow \mathbb{R} P^{2}$.)

\section{Appendix: used facts on compact Lie groups}

Recall that the centralizer of an element $h \in G$ is the subset of $G$ of the elements that leave $h$ fixed,

$$
Z(h)=\left\{g \in G: g h g^{-1}=h\right\} .
$$

It is immediate to prove that the centralizer is a subgroup. The normalizer of a subgroup $H$ of $G$ is the set of elements that leave $H$ fixed

$$
N(H)=\left\{g \in G: g H g^{-1}=H\right\} .
$$

$N(H)$ is a subgroup of $G$ which contains $H$ and is the biggest subgroup of $G$ in which $H$ is a normal subgroup.

Given a compact group $G$, and $h \in G$, the subgroup $Z(h)$ is a compact subgroup of $G$ and, if Abelian, is a maximal torus. When this happens, $h$ is called regular, $Z(h) \cong T$ is called a Cartan subgroup, and its Lie algebra $\mathfrak{t}$ is called a Cartan subalgebra. The dimension $r$ of a Cartan subgroup is the same for all Cartan subgroups and is called the rank of the group $G$. All Cartan subgroups are conjugate (see page 159 in [3]).

Consider now a Cartan subgroup $T$ and its Cartan subalgebra $\mathfrak{t}$. The normalizer of $T$ in $G$ is a finite extension of $T$ (see page 158 in [3]), and the finite group $W=N(T) / T$ is called the Weyl group.

The group $N(T)$ acts by conjugation on $T$, action that obviously defines an action of the Weyl group on $T$ and, by linearization, an action on the Cartan algebra $\mathfrak{t}$. The Weyl group action on $\mathfrak{t}$ is a group of reflections (see page 192 in [3]), each reflection in the Weyl group fixes an hyperplane, called a Weyl wall.

This fact, together with the fact that every element of $G$ belongs to at least one Cartan subgroup, implies that the set of regular elements is an open dense set. To complete the picture 
we observe the trivial fact that the exponential of elements in a Weyl wall give precisely the elements which belong to more than a maximal torus or, in other words, those whose centralizer is a bigger, non-commutative subgroup of $G$.

\section{Acknowledgements}

The authors thank the Bernoulli Center (EPFL, Lausanne) for its hospitality during the 2004 program Geometric Mechanics and Its Applications, where the biggest part of this work was done, and Hans Duistermaat for some enlightening conversations on these topics.

\section{References}

[1] Blaom A.D., A geometric setting for Hamiltonian perturbation theory, Mem. Amer. Math. Soc. 153 (2001), $1-112$.

[2] Bogoyavlenskij O.I., Extended integrability and bi-Hamiltonian systems, Comm. Math. Phys. 196 (1998), $19-51$.

[3] Bröcker T., tom Dieck T., Representations of compact Lie groups, Graduate Texts in Mathematics, Vol. 98, Springer-Verlag, New York, 1995.

[4] Cushman R., Duistermaat J.J., Non-Hamiltonian monodromy, J. Differential Equations 172 (2001), 42-58.

[5] Cushman R., Duistermaat H., Snyaticky J., Non-holonomic systems, in preparation.

[6] Duistermaat J.J., On global action-angle coordinates, Comm. Pure Appl. Math. 33 (1980), 687-706.

[7] Fassò F., Superintegrable Hamiltonian systems: geometry and perturbations, Acta Appl. Math. 87 (2005), 93-121.

[8] Fassò F., Giacobbe A., Sansonetto N., Periodic flow, rank-two Poisson structures, and nonholonomic mechanics, Regular Chaotic Mech. 19 (2005), 267-284.

[9] Fedorov Yu.N., Systems with an invariant measure on Lie groups, in Hamiltonian Systems with Three or More Degrees of Freedom (1995, S'Agarò), NATO Adv. Sci. Inst. Ser. C Math. Phys. Sci., Vol. 533, Kluwer, Dordrecht, 1999, 350-356.

[10] Field M., Local structure of equivariant dynamics, in Singularity Theory and Its Applications, Part II (1988/1989, Coventry), Lecture Notes in Math., Vol. 1463, Springer, Berlin, 1991, 142-166.

[11] Fomenko A.T., Trofimov V.V., Integrable systems on Lie algebras and symmetric spaces, Advanced Studies in Contemporary Mathematics, Vol. 2, Gordon and Breach Science Publishers, New York, 1988.

[12] Hermans J., Rolling rigid bodies with and without symmetries, PhD Thesis, University of Utrecht, 1995.

[13] Hermans J., A symmetric sphere rolling on a surface, Nonlinearity 8 (1995), 493-515.

[14] Karasev M.V., Maslov V.P., Nonlinear Poisson brackets. Geometry and quantization, Translations of the $A M S$, Vol. 119, AMS, Providence, R.I., 1993.

[15] Marsden J.E., Montgomery R., Ratiu T., Reduction, symmetry, and phases in mechanics, Mem. Amer. Math. Soc. 88 (1990), no. 436.

[16] Meigniez G., Submersion, fibrations and bundles, Trans. Amer. Math. Soc. 354 (2002), 3771-3787.

[17] Mischenko A.S., Fomenko A.T., Generalized Liouville method of integration of Hamiltonian systems, Funct. Anal. Appl. 12 (1978), 113-121.

[18] Routh E.J., Treatise on the dynamics of a system of rigid bodies (advanced part), Dover, New York, 1955.

[19] Zenkov D.V., The geometry of the Routh problem, J. Nonlinear Sci. 5 (1995), 503-519.

[20] Zung N.T., Torus action and integrable systems, in Topological Methods in the Theory of Integrable Systems, Editors A.V. Bolsinov, A.T. Fomenko and A.A. Oshemkov, Cambridge Scientific Publications, 2006, 289328 , math.DS/0407455. 